\title{
DIELECTRIC PROPERTIES OF PIEZOELECTRIC POLYIMIDES
}

\section{Z. OUNAIES ${ }^{1}$, J. A. YOUNG ${ }^{2}$, J. O. SIMPSON ${ }^{3}$, B. L. FARMER ${ }^{2}$}

${ }^{1}$ National Research Council, NASA Langley Research Center, Hampton, VA 23681

${ }^{2}$ University of Virginia, Department of Materials Science and Engineering, Charlottesville, VA 22903

${ }^{3}$ Composites and Polymers Branch, NASA Langley Research Center, Hampton, VA 23681

\begin{abstract}
Molecular modeling and dielectric measurements are being used to identify mechanisms governing piezoelectric behavior in polyimides such as dipole orientation during poling, as well as degree of piezoelectricity achievable. Molecular modeling on polyimides containing pendant, polar nitrile $(\mathrm{CN})$ groups has been completed to determine their remanent polarization. Experimental investigation of their dielectric properties evaluated as a function of temperature and frequency has substantiated numerical predictions. With this information in hand, we are then able to suggest changes in the molecular structures, which will then improve upon the piezoelectric response.
\end{abstract}

\section{INTRODUCTION}

This investigation is motivated by NASA's interest in developing high performance piezoelectric polymers for a variety of high temperature aerospace applications. Reported herein are numerical calculations and experimental results which are used to characterize and understand the poling of a piezoelectric, nitrile-substituted aromatic polyimide [1,2]. Molecular modeling provides fundamental understanding of the polyimide's response to temperature and electric field. The experimental studies are used to evaluate the accuracy of the model.

Molecular structures of the polyimides investigated are given in Figure 1 below.

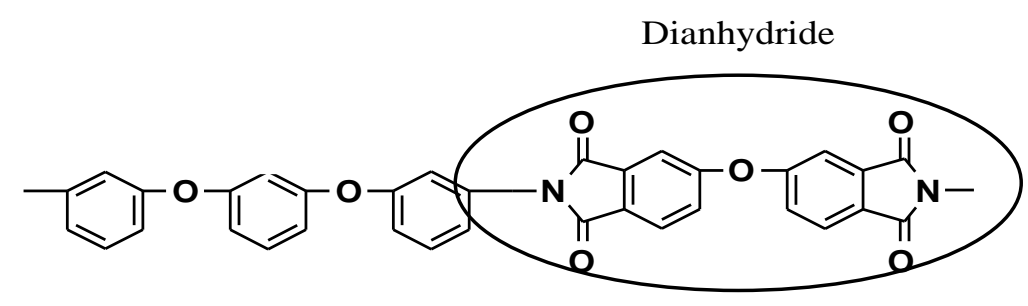

APB/ODPA

$\mathrm{T}_{\mathrm{g}}=185^{\circ} \mathrm{C}$

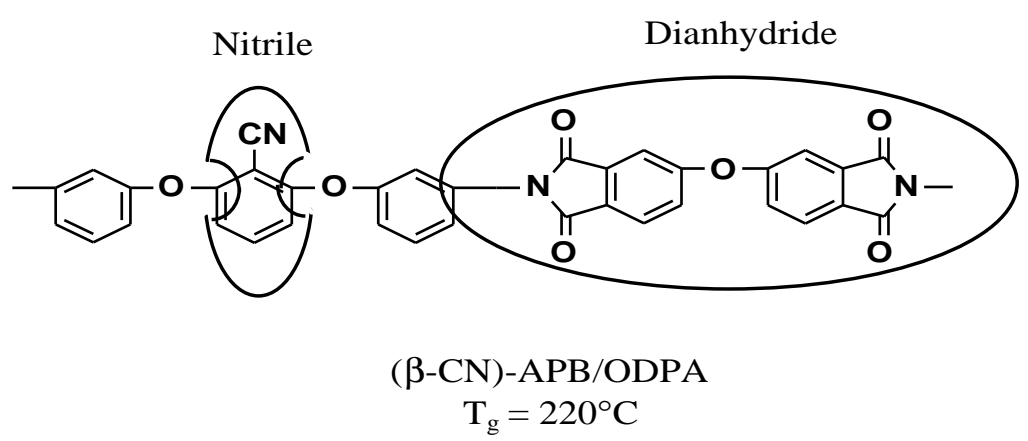

Figure 1. Molecular structures of polyimides studied. 


\section{METHODS}

In order to induce a piezoelectric response in the polyimide systems shown above, they are poled by applying a strong electric field $\left(E_{p}\right)$ at an elevated temperature $\left(T_{p} \geq T_{g}\right)$ which produces orientation of the molecular dipoles. Partial retention of this orientation is achieved by lowering the temperature below $\mathrm{T}_{\mathrm{g}}$ in the presence of $\mathrm{E}_{\mathrm{p}}$. This is known as orientation polarization . In order to maximize piezoelectricity, the remanent polarization $\left(\mathrm{P}_{\mathrm{r}}\right)$ must be maximized. $\mathrm{P}_{\mathrm{r}}$ is related to the poling field by

$$
\mathrm{P}_{\mathrm{r}}=\varepsilon_{\mathrm{o}} \Delta \varepsilon \mathrm{E}_{\mathrm{p}}
$$

where $\varepsilon_{\mathrm{o}}$ is the permittivity of space, and $\Delta \varepsilon$ is the change in dielectric constant upon traversing the glass transition. As pointed out by Furukawa [3], $\Delta \varepsilon$ is the parameter of greatest interest in designing amorphous polymers with large piezoelectric activity. Due to dielectric breakdown of the polymeric materials, $\mathrm{E}_{\mathrm{p}}$ is limited to approximately $100 \mathrm{MV} / \mathrm{m}$. Hence, molecular design must be implemented to increase the $\Delta \varepsilon$ and consequently $\mathrm{P}_{\mathrm{r}}$.

\section{Computational}

Molecular modeling of high temperature polyimides is done within the BIOSYM molecular modeling package. Initial quantum mechanical calculations using MOPAC have been done on segments of the polyimide to assign force field parameters and partial atomic charges for the polymer. The computational model used in the molecular dynamic simulations is a chain of five repeat units within a three-dimensionally periodic cell. The cell is 'plated' with dummy atoms upon which partial charges are placed to emulate the applied electric field in the z-direction [4].

Molecular dynamics is employed to solve the classical equations of motion for all of the atoms comprising the polymer. A dynamic picture of the system is obtained as a function of temperature, applied electric field and time. Experimental polymer relaxation times tend to be quite long in the vicinity of $\mathrm{T}_{\mathrm{g}}$ (seconds or minutes). By using an artificially high temperature in the simulation, however, the polarization is seen to reach a steady-state value in $50 \mathrm{ps}$. An analysis of the relaxation behavior will be reported elsewhere [4]. The time/temperature relationship is of the familiar Arrhenius form presented in detail by [5],

$$
\tau=\left[3 \varepsilon_{\mathrm{s}} /\left(2 \varepsilon_{\mathrm{s}}+\varepsilon_{\infty}\right)\right][\mathrm{h} / \mathrm{k} \mathrm{T}][\exp (\Delta \mathrm{F} / \mathrm{kT})]
$$

The magnitude of the electric field is also increased in order to maintain the population density of dipoles (i.e. $\mathrm{m} \mathrm{E} / \mathrm{kT}$ ). Molecular dynamics is performed at $1700{ }^{\circ} \mathrm{C}$ for $50 \mathrm{ps}$ in the

absence of a poling field in order to obtain starting conformations. A poling field of $700 \mathrm{MV} / \mathrm{m}$ is then applied for $200 \mathrm{ps}$. The polarization is then frozen in by running molecular dynamics for 200 ps at $25^{\circ} \mathrm{C}$. This yields to the steady-state value of the remanent polarization.

\section{$\underline{\text { Experimental }}$}

Experimental studies are made on films synthesized at NASA Langley Research Center. Silver electrodes are evaporated on both sides of the polymer. The dielectric constant and loss as a function of temperature and frequency are evaluated on unpoled APB/ODPA and ( $\beta-C N)$ APB/ODPA samples using a Hewlett Packard 4192A Impedance Analyzer. The measurements are done at temperatures ranging from ambient to $300^{\circ} \mathrm{C}$ at $5 \mathrm{~Hz}, 10 \mathrm{~Hz}, 100 \mathrm{~Hz}$ and $1 \mathrm{kHz}$.

Poling of the polyimide samples is done in an oil bath at $\mathrm{T}_{\mathrm{p}}=\mathrm{T}_{\mathrm{g}}+4^{\circ} \mathrm{C}$ at $\mathrm{E}_{\mathrm{p}}=50,80$, and $100 \mathrm{MV} / \mathrm{m}$ for $t_{p}=30 \mathrm{~min}$. To evaluate the $P_{r}$ in the poled samples, a thermally stimulated current (TSC) analysis is used[6]. A Keithley 6517 electrometer is connected to the sample to record the 
short-circuit current while the sample is heated at a constant rate of $1{ }^{\circ} \mathrm{C} / \mathrm{min}$ from room temperature to $\mathrm{T}_{\mathrm{g}}+20^{\circ} \mathrm{C}$. The current is created as the material depolarizes with increasing temperature.

\section{RESULTS}

Using the molecular modeling outlined previously, three cells of ( $\beta$-CN)-APB/ODPA are independently built and poled at $150 \mathrm{MV} / \mathrm{m}$. The polarization of each cell is then calculated using the atomic charges and positions during the molecular dynamics run. As seen in Figure 2 the final polarization in the direction of the applied field, the $\mathrm{z}$ direction, is independent of the initial starting conformation. $\Delta \varepsilon$ is obtained using equation 1. The average value of $\Delta \varepsilon$ is $17.8 \pm 1.1$ for ( $\beta$ $\mathrm{CN})-\mathrm{APB} / \mathrm{ODPA}$.

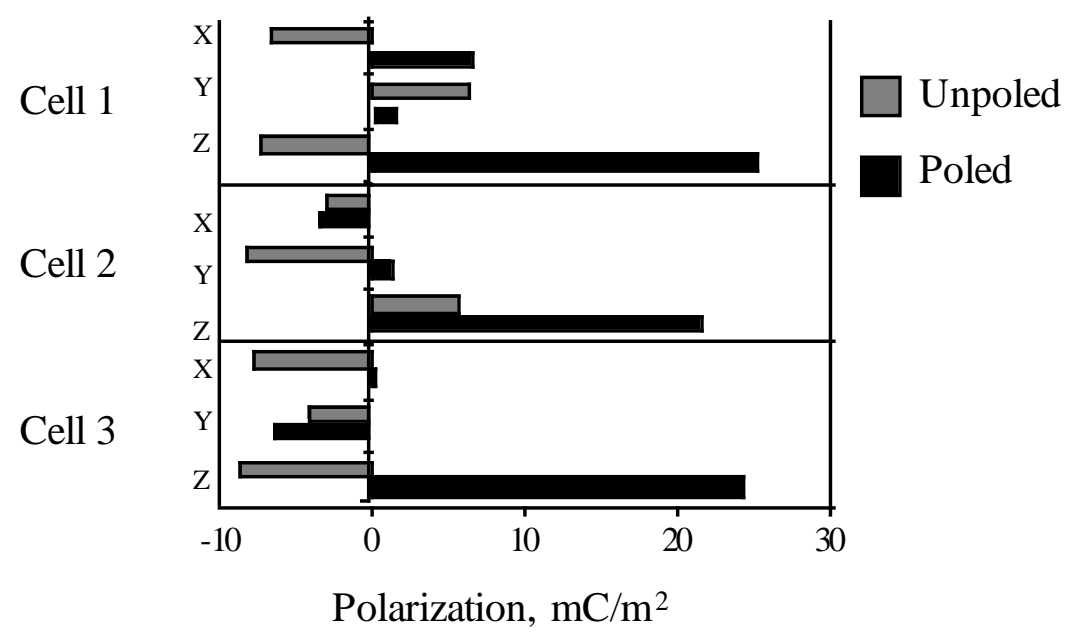

Figure 2. Polarization along principle axes for the three starting configurations.

The contributions of specific dipoles along the polymer chain (Figure 3) to the total polarization of the cell are examined. The ability of nitrile substituents to create large polarizations in polymeric systems has been previously demonstrated $[7,8]$. On average, the nitrile substituent constitutes only $48 \%$ of the total polarization. The dianhydride also provides significant polarization, constituting about $39 \%$ of the total.

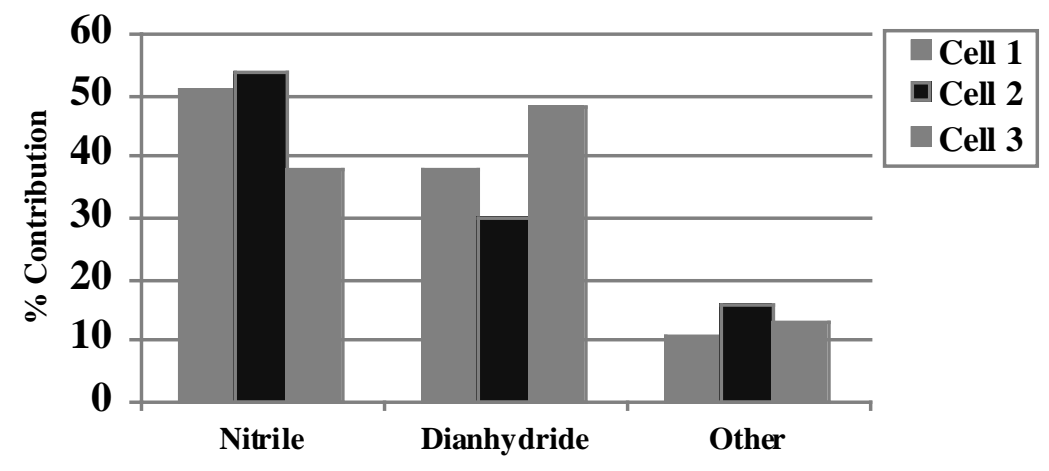

Figure 3. Segmental contribution to polarization.

The unsubstituted APB/ODPA is then modeled. Assuming that the polar segments of the polymer contribute to the polarization independently, APB/ODPA is expected to have a polarization of $48 \%$ less than that of $(\beta-C N)-A P B / O D P A$, i.e. $\Delta \varepsilon=9.2$. Molecular simulations of APB/ODPA actually yield $\Delta \varepsilon=7.7 \pm 1.6$, in excellent agreement with the expected value. Examination of the 
molecular dynamics trajectories shows that the majority of the polarization, i.e. $81 \%$, is now due to the dianhydride portion of the polymer.

Figure 4 shows the dielectric constant and loss as a function of frequency and temperature for the $(\beta-\mathrm{CN})$-APB/ODPA polymer. In analyzing the data, care must be taken to separate the electrical conduction effects from dipolar reorientation. Inspection of Figure 4 reveal that the dielectric properties are reaching a plateau at temperatures around $300^{\circ} \mathrm{C}$. It was observed that conductivity effects and cross-linking of the substituents occur at temperatures higher than $300^{\circ} \mathrm{C}$ as evidenced by the increase in the dielectric loss. As the frequency decreases, the onset of the dielectric relaxation occurs at a lower temperature. The value of $\Delta \varepsilon$ also increases with decreasing frequency because the molecular dipoles are more able to follow the low frequency signal. This indicates that the lowest measurement frequency (i.e. $5 \mathrm{~Hz}$ ) most closely approximates the dc poling conditions. As shown in Table I, the $\Delta \varepsilon$ at $5 \mathrm{~Hz}$ is in excellent agreement with computational predictions.

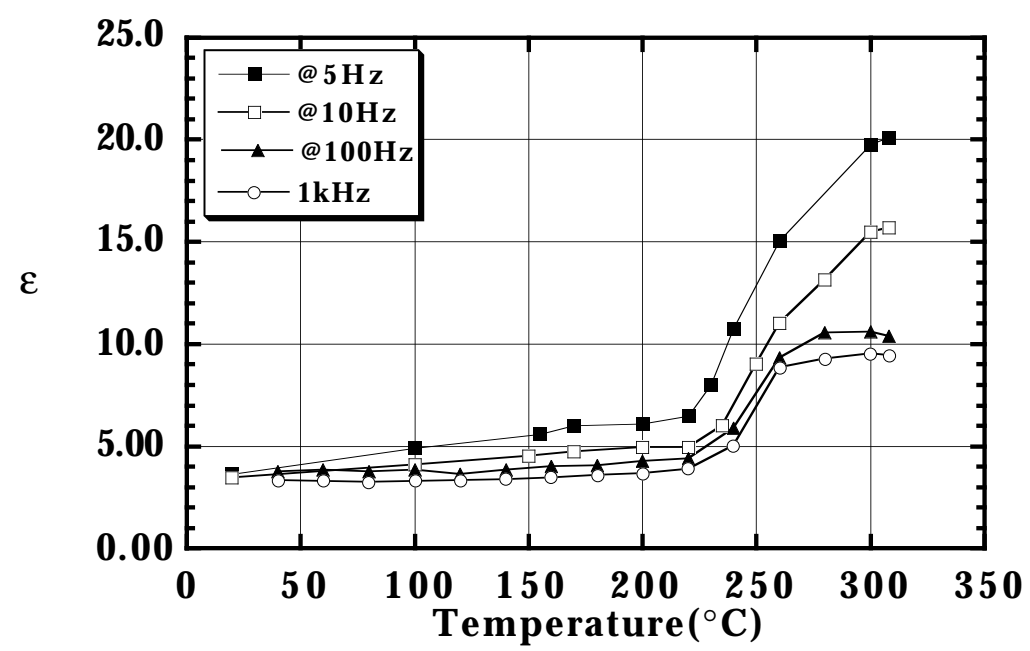

Figure 4a. Dielectric constant as a function of temperature and frequency for the $(\beta-\mathrm{CN})$ -

APB/ODPA polymer.

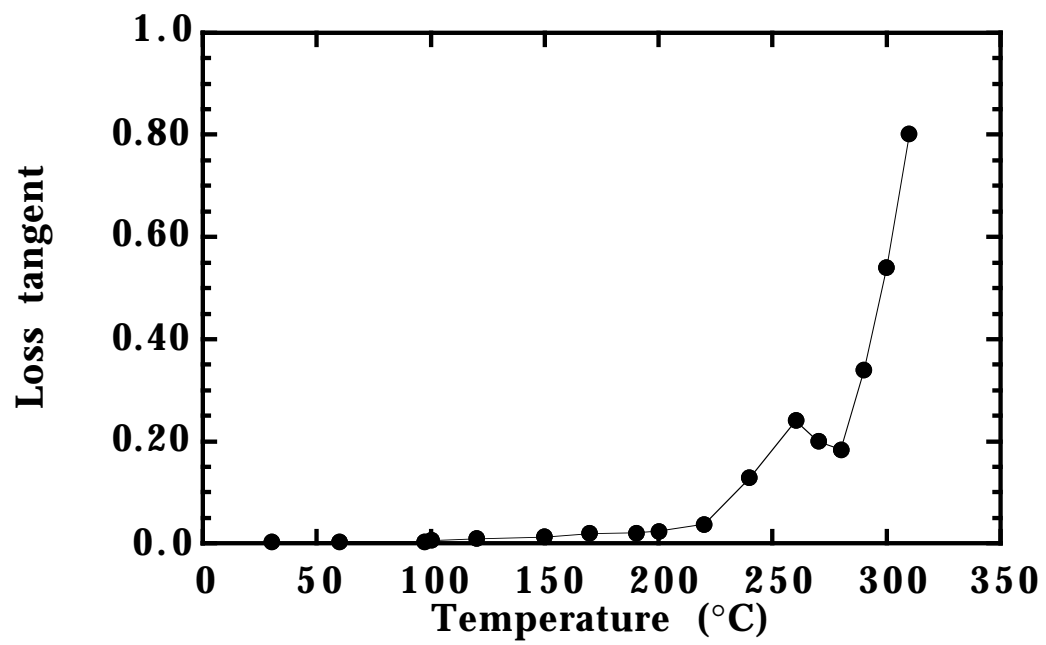

Figure $4 \mathrm{~b}$. Dielectric loss as a function of temperature at $1 \mathrm{kHz}$ for the $(\beta-\mathrm{CN})-\mathrm{APB} / \mathrm{ODPA}$ polymer. 
Table I. Comparison between computed and measured $\Delta \varepsilon$

\begin{tabular}{|c|c|c|c|}
\hline & $\Delta \varepsilon_{10 \mathrm{H}}$ & $\Delta \varepsilon_{\mathrm{sH}}$ & $\Delta \varepsilon_{\text {camn }}$ \\
\hline$(\beta-\mathrm{CN})-\mathrm{APB} / \mathrm{ODPA}$ & $\mathbf{1 1 . 5} \pm \mathbf{0 . 6}$ & $\mathbf{1 6} \pm \mathbf{1 . 3}$ & $\mathbf{1 7 . 8} \pm \mathbf{1 . 1}$ \\
\hline $\mathrm{APB} / \mathrm{ODPA}$ & $\mathbf{4 . 2} \pm \mathbf{0 . 5}$ & $\mathbf{5 . 7} \pm \mathbf{1 . 5}$ & $\mathbf{7 . 7} \pm \mathbf{1 . 6}$ \\
\hline
\end{tabular}

An example of the spectrum obtained from the TSC analysis is shown in Figure 5. It is described by a single current peak that correlates well with the glass transition temperature of the material as measured by DSC. By integrating the current/temperature curve as a function of time, the remanent polarization is obtained. Figure 6 shows the value of $\mathrm{P}_{\mathrm{r}}$ as a function of applied field. As expected, there is a linear correlation between the poling field and the resultant $\mathrm{P}_{\mathrm{r}}$. Once the poling field is removed, a certain amount of dipole relaxation is expected, and this is seen by the difference between $\mathrm{P}_{\mathrm{r}}$ computed from equation 1 and $\mathrm{P}_{\mathrm{r}}$ obtained from TSC.

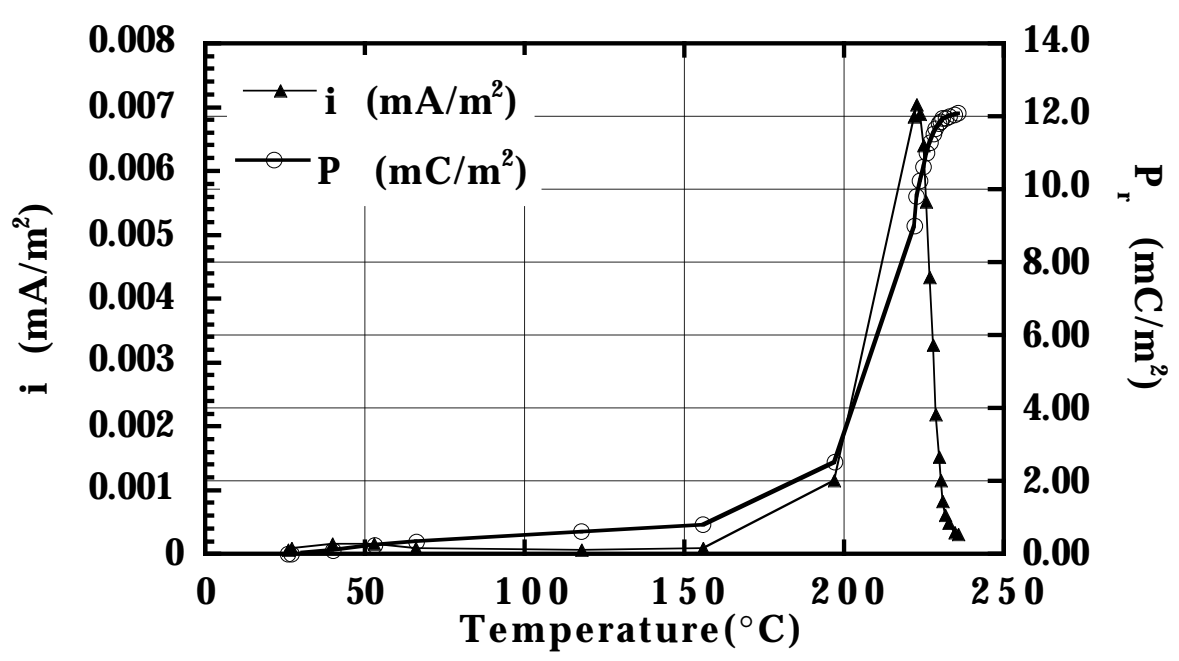

Figure 5. Thermally stimulated current and remanent polarization for $(\beta-\mathrm{CN})-\mathrm{APB} / \mathrm{ODPA}$. $\mathrm{E}_{\mathrm{p}}=100 \mathrm{MV} / \mathrm{m}, \mathrm{T}_{\mathrm{p}}=223^{\circ} \mathrm{C}, \mathrm{t}_{\mathrm{p}}=30 \mathrm{~min}$

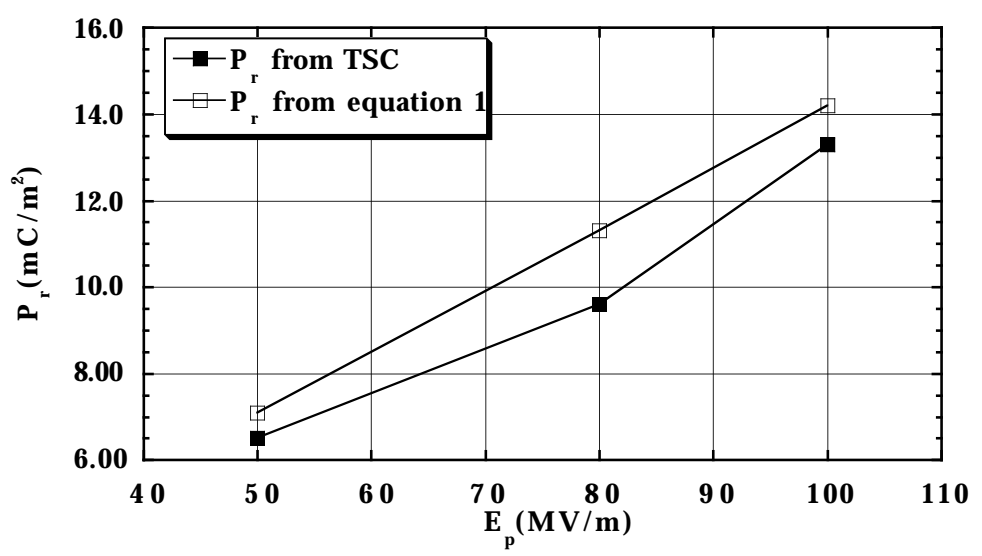

Figure 6. Remanent polarization as a function of poling field. 


\section{CONCLUSIONS}

In this analysis, a high $\mathrm{T}_{\mathrm{g}}$, amorphous polymer is studied using both modeling and experimental methods. The goal of both methods is to evaluate and optimize the remanent polarization $\mathrm{P}_{\mathrm{r}}$ and hence the piezoelectric behavior. The dipole moment of the nitrile substituent as well as the dianhydride contribute significantly to the polarization and piezoelectric response of the polyimide. Both the dielectric measurements and the TSC technique yield similar $\mathrm{P}_{\mathrm{r}}$.

The computational and experimental results for the APB/ODPA systems are in good agreement. Through the computational modeling, we have succesfully developed a tool to guide the development of polymeric materials with improved piezoelectric responses. Such tools could reduce the time and cost of material production.

\section{ACKNOWLEDGEMENTS}

The authors gratefully acknowledge the technical insight of Drs. Jeffrey Hinkley and Terry St. Clair of NASA Langley Research Center. Also acknowledged is Dr. Catherine Fay of the National Research Council for the synthesis of polyimide films.

\section{REFERENCES}

1. J. O. Simpson. S. S. Welch and T. L. St. Clair, in Electrical, Optical and Magnetic Properties of Organic Solid State Materials III, edited by A. K-Y. Jen, C. Y-C. Lee, L. R. Dalton, M. F. Rubner and G. E. Wnek (Mater. Res. Soc. Proc. 143, Boston, MA 1995), p.351-356.

2. J. A. Hinkley, High Performance Polymers 8, (1996).

3. T. Furukawa, IEEE Trans. Elec. Ins. 24, 375 (1989).

4. J. A. Young, (to be published).

5. B. Hilezer and J. Mlecki, Electrets, Polish Scientific Publishers, Warszawa, 1986.

6. J. P. Ibar, P. Denning, T. Thomas, A. Bernes, C. de Goys, J. R. Saffell, P. Jones and C. Lacabanne, in Polymer Characterization: Physical Property, Spectroscopy, and Chromotographic methods, edited by C. D. Craver and T. Provder (Adv. Chem. Series 227, 1990), p. 167-190.

7. S. Tasaka, T. Toyama and N. Inagaki, Jpn. J. Appl. Phys. 33, 5838 (1994).

8. S. Tasaka, N. Inagaki, T. Okutani and S. Miyata, Polymer 30, 1639 (1989). 